\title{
A study of airfoil parameterization, modeling, and optimization based on the computational fluid dynamics method*
}

\author{
Tian-tian ZHANG, Wei HUANG ${ }^{\dagger}$, Zhen-guo WANG, Li YAN \\ (Science and Technology on Scramjet Laboratory, National University of Defense Technology, Changsha 410073, China) \\ ${ }^{\dagger}$ E-mail: weihuang@nudt.edu.cn \\ Received Nov. 22, 2015; Revision accepted Jan. 20, 2016; Crosschecked July 24, 2016
}

\begin{abstract}
An excellent airfoil with a high lift-to-drag ratio may decrease oil consumption and enhance the voyage. Based on NACA 0012, an improved airfoil is explored in this paper. The class/shape function transformation has been proved to be a good method for airfoil parameterization, and in this paper it is modified to improve imitation accuracy. The computational fluid dynamics method is applied to obtain numerically the aerodynamic parameters of the parameterized airfoil, and the result is proved credible by comparison with available experimental data in the open literature. A polynomial-based response surface model and the uniform Latin hypercube sampling method are employed to decrease computational cost. Finally, the nonlinear programming by quadratic Lagrangian method is utilized to modify the multi-island genetic algorithm, which has an improved optimization effect than the method used on its own. The obtained result shows that the modified class/shape function transformation method produces a better imitation of an airfoil in the nose and tail regions than the original method, and that it will satisfy the tolerance zone of the model in a wind tunnel. The response surface model based on the uniform Latin hypercube sampling method gives an accurate prediction of the lift-to-drag ratio with changes in the design variables. The numerical result of the flow around the airfoil shows reasonable agreement with the experimental data graphically and quantitatively. Ultimately, an airfoil with better capacity than the original one is acquired using the multi-island genetic algorithm based nonlinear programming by quadratic Lagrangian optimization method. The pressure contours and lift-to-drag ratio along with the attack angle have been compared with those of the original airfoil, and the results demonstrate the strength of the optimized airfoil. The process for exploring an improved airfoil through parameterization to optimization is worth referencing in future work.
\end{abstract}

Key words: Class/shape function transformation (CST), Parameterization, Numerical simulation, Response surface model, Optimization, Airfoil design http://dx.doi.org/10.1631/jzus.A1500308

CLC number: V22

\section{Introduction}

In modern society, flying by air has become a more and more popular part of people's lives. With the development of the aviation industry, environmental aircraft with low flight costs have attracted increasing attention worldwide. Improving the ca-

\footnotetext{
¿ Corresponding author

* Project supported by the National Natural Science Foundation of China (No. 11502291), and the Fund of Outstanding Doctoral Dissertation from the Ministry of Education of China (No. 201460) (D) ORCID: Wei HUANG, http://orcid.org/0000-0001-9805-985X (C) Zhejiang University and Springer-Verlag Berlin Heidelberg 2016
}

pacity of aircraft is essential work for their designers. According to the voyage equation, the lift-to-drag ratio of an aircraft is very important for enhancing the voyage in terms of keeping the oil costs low (Huang et al., 2012c), and this relates to the environment surrounding the aircraft. The choice of airfoil is of great importance in aircraft design; thus, it is very important to discover the characteristics of airfoils with different shapes. With the development of computing techniques, the numerical method is becoming a significant technique in the fields of aviation and aerospace vehicle design (Huang, 2015). Therefore, an accurate approach for transforming the 
geometric configuration to parameters that can be identified by computers is of great importance. Yu et al. (2003) reviewed the existing method of airfoil parameterization and proposed a novel method based on the general fifth-order parametric spline. This method can work with nearly any airfoil using 7-13 control points, but the control point optimization process is time consuming. The class/shape function transformation (CST) method (Kulfan, 2007) is a novel parameterization method which can model a wide array of smooth geometries with a small number of equations and parameters (Kevin and David, 2009). It has been modified as class/shape refinement transformation (CSRT) by Straathof and van Tooren (2012) to improve local matching ability, but the number of variables increases for that method. A numerical method has been applied to obtain information about the flow field around airfoils by Nejat et al. (2014), but the calculation program used, namely XFOIL, can deal with only flow with no viscosity, and they failed to discuss the precision that was calculated; the numerical results often failed to compare with the experimental data. In the optimization process, an increase in the number of design variables will lead to enormous computational costs; thus, a surrogate model and a reasonable sampling method are required to replace the numerical process. Luo and $\mathrm{Lu}$ (2014) compared polynomial regression $(\mathrm{PR})$, radial basis function artificial neural networks (RBFANN), and kriging methods for simulating a multiphase problem, which proved the advantage of establishing a surrogate model before optimization. The accuracy of surrogate models for a particular problem should be checked because different models may have different performances. Global optimization algorithms such as the multi-island genetic algorithm (MIGA) (Zhao et al., 2015) and optimal gradient methods such as nonlinear programming by quadratic Lagrangian (NLPQL) have been presented recently, and their capacities for airfoil optimization have been compared in this paper. Ma et al. (2015) optimized the aerodynamic shape of a hypersonic lifting body using a multi-objective evolutionary algorithm based on a decomposition (MOEA/D) method, which was in turn based on the kriging model, but the shape function of the CST method they used was omitted in optimization, so not all configurations may have been presented.
This paper has improved the CST method by redistributing the control points without changing the number of variable parameters. The computational fluid dynamics (CFD) approach has been proved credible, and the result has been compared with the experimental data graphically and quantitatively. The optimization methods NLPQL and MIGA are combined in an airfoil optimization process based on the response surface model, and the result shows better capacity than that obtained by the optimization method on its own. This paper illustrates the processes of parameterization, sampling, modeling, and optimization of an airfoil based on the CFD method.

\section{Parameterization method}

Low-speed airfoils typically have a configuration with a round nose and a sharp tail. Traditional methods, such as the polynomial fitting method, the spline method, Hicks-Henne, and the parametric section method (PARSEC), describe their basic profile. However, the weaknesses in accuracy and robustness of these methods are exposed when the number of describing parameters is limited (Zhang et al., 2014). Kulfan (2007) presented a novel two-step airfoil fitting technique, namely CST, which is defined as

$$
\varsigma(\varphi)=C_{N_{2}}^{N_{1}}(\varphi) \times S(\varphi),
$$

where $\varphi$ stands for $x / c$, and $c$ is the chord length. Thus, $\varphi$ is the normalized coordinate in the $x$ direction. $C_{N_{2}}^{N_{1}}(\varphi)$ is the class function, and $S(\varphi)$ stands for shape function, which may together describe the original curve. The basic classification of the airfoil curve is determined by the class function, which is controlled by two parameters, namely $N_{1}$ and $N_{2}$ :

$$
C_{N_{2}}^{N_{1}}(\varphi)=\varphi^{N_{1}}(1-\varphi)^{N_{2}}
$$

Morris et al. (2014), Nejat et al. (2014), and Su et al. (2015) have showed the shape of curves for different values of $N_{1}$ and $N_{2}$. They also claimed that when describing an airfoil, the values we normally use are $N_{1}=0.5$ and $N_{2}=1$. Bernstein's polynomial is often used as the basic shape function, which is described as 


$$
S(\varphi)=\sum_{i=0}^{n} B_{i} \cdot\left(\begin{array}{l}
n \\
i
\end{array}\right) \varphi^{i}(1-\varphi)^{n-i}
$$

where $n$ stands for the order of polynomial, and $B_{i}$ is Bernstein's coefficient, which has $n+1$ in total. It also implies that $n+1$ control points will be needed to modify the shape function. Different values of $B_{i}$ will represent different curves; thus, $B_{i}$ is the lynchpin for airfoil fitting and optimization. We can conclude from Eq. (3) that each $B_{i}$ coefficient in Bernstein's polynomial will produce global variance of the curve. To settle this problem, Straathof and van Tooren (2011; 2012) put forward a refined CST method (CSRT) using a B-spline. The B-spline function is employed to modify the curve locally, based on CST. This method has been applied successfully in the parameterization of 2D airfoils, and it was also expanded to 3D parameterization. Although the CSRT parameterization method can improve airfoil imitation accuracy in the nose and the tail areas, this method will increase the number of design variables, and it will dramatically increase the computation cost and reduce the efficiency, especially during the optimization process. Sobieczky (1999) claimed that it would satisfy the tolerance zone of the model in a wind tunnel when the fitting error of airfoil parameterization is below 0.0007 . Therefore, it is unnecessary to add design variables when the accuracy condition is satisfied.

To check the strength of CST in airfoil parameterization, NACA 1412 is utilized as the basic curve to be fitted using the CST and polynomial methods. The fifth-order form was applied in both of the methods, and the fitting results are shown in Figs. 1 and 2. Fig. 1 illustrates the matching error distribution with the fifth-order CST method. It can be found that the maximum error is below 0.0005 , and it satisfies the tolerance requirement proposed by Sobieczky (1999). However, the matching error of the fifth-order polynomial method illustrated in Fig. 2 has an order of magnitude of $10^{-3}$, which is out of the tolerance zone. Thus, it cannot match the airfoil configuration accurately.

An airfoil with no chamber such as NACA 0012 has a symmetric surface and a more regular shape. It was parameterized by CST and the polynomial method from the first order to the fifth order. As shown in Figs. 3 and 4, the matching errors of the upper surface for different matching orders are
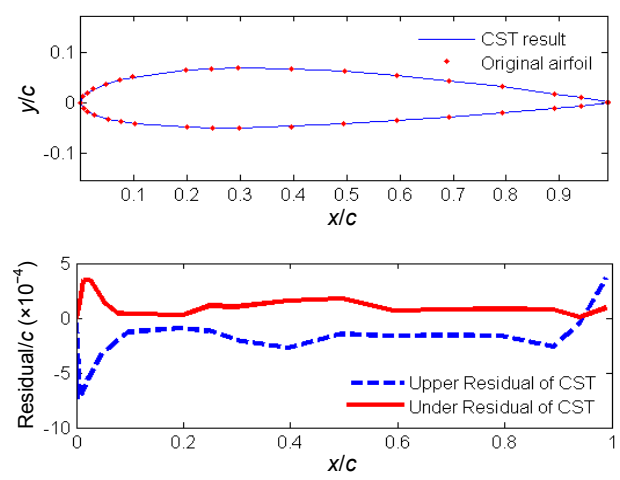

Fig. 1 Comparison between the NACA 1412 airfoil and the fifth-order CST imitation result
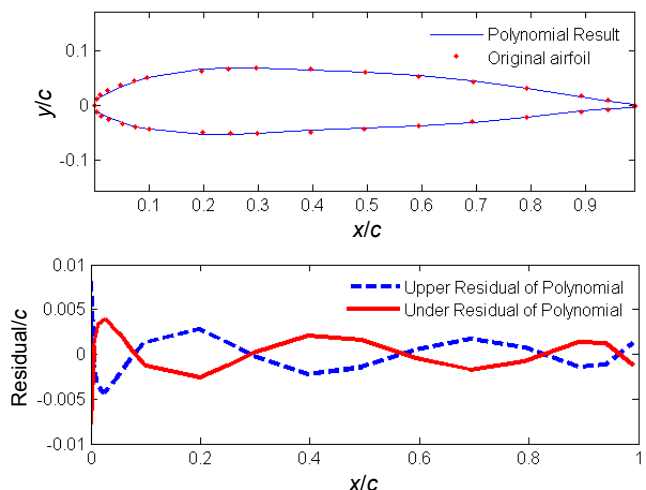

Fig. 2 Comparison between the NACA 1412 airfoil and the fifth-order polynomial imitation result

compared. The standard deviations of the matching residual (SR) for different orders are calculated by

$$
\mathrm{SR}=\sqrt{\frac{\sum_{i=1}^{n_{\mathrm{s}}}[Y(i) / c-y(i) / c]^{2}}{n_{\mathrm{s}}}},
$$

where $Y(i)$ stands for the original ordinate of the samples, while $y(i)$ is the matching ordinate; $n_{\mathrm{s}}$ is the number of samples. The values tell us that both of the parametric methods have smaller standard deviations for matching error as the matching order increases. However, CST is far more accurate than the polynomial method when the matching order is fixed. Moreover, as for a simple airfoil, such as NACA 0012, CST has a precision below 0.0004 when the matching order is just 1 , and it is enough to satisfy the tolerance requirement. It implies that two design variables are enough to describe this airfoil using the CST method. In comparison, the polynomial method 
cannot describe the airfoil accurately with few variables. As a result, CST is a good choice for the parameterization and optimization of airfoils.
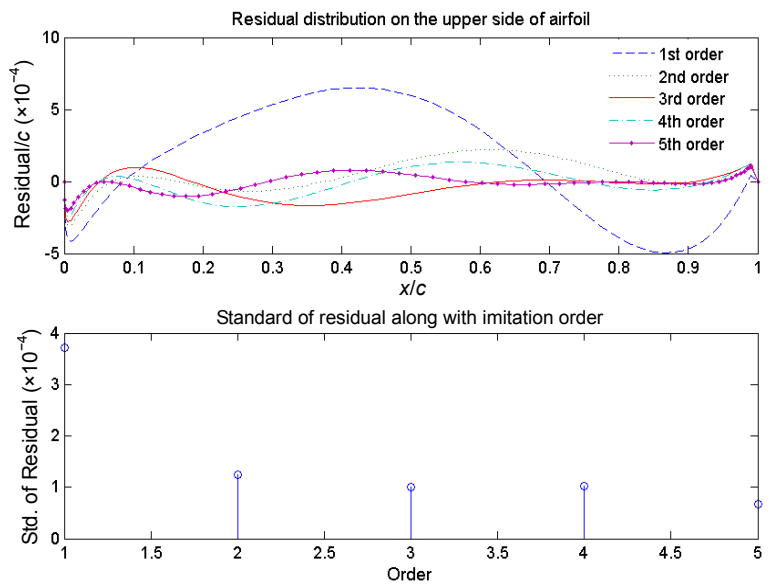

Fig. 3 CST imitation residual for different orders for the NACA 0012 airfoil
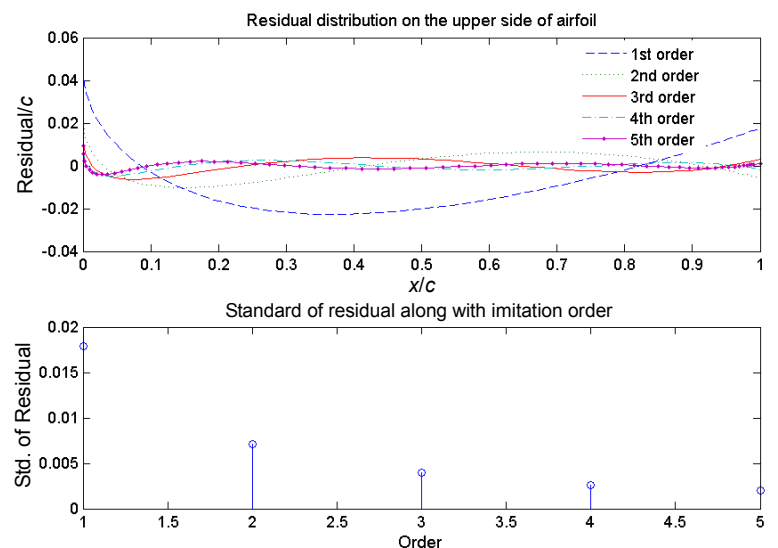

Fig. 4 Polynomial imitation residual for different orders for the NACA 0012 airfoil

Acquiring a value for Bernstein's coefficient in the shape function is the most significant step in parameterization by the CST method. The optimization method and the method used to calculate the coefficient matrix are normally used to acquire Bernstein's coefficient. The optimization method will obtain the coefficient by minimizing the standard deviations of the matching error for a given order. This method has a great ability for adaption and high accuracy, which can also satisfy the calculation for higher orders. However, when the number of design variables grows, computing cost will increase dramatically. The choice of optimization method will also influence the veracity of the result. The method for calculating the coefficient matrix involves choosing several control points $\left[\varphi_{j}, y\left(\varphi_{j}\right)\right]$ with the number equal to the fitting order.

Assuming that

$$
M_{i}\left(\varphi_{j}\right)=C\left(\varphi_{j}\right) \cdot\left(\begin{array}{c}
n \\
i
\end{array}\right) \varphi_{j}^{i}\left(1-\varphi_{j}\right)^{n-i}
$$

Thus,

$$
\left[\begin{array}{cccc}
M_{0}\left(\varphi_{0}\right) & M_{1}\left(\varphi_{0}\right) & \cdots & M_{n}\left(\varphi_{0}\right) \\
M_{0}\left(\varphi_{1}\right) & M_{1}\left(\varphi_{1}\right) & \cdots & M_{n}\left(\varphi_{1}\right) \\
\vdots & \vdots & & \vdots \\
M_{0}\left(\varphi_{n}\right) & M_{1}\left(\varphi_{n}\right) & \cdots & M_{n}\left(\varphi_{n}\right)
\end{array}\right]\left[\begin{array}{c}
B_{0} \\
B_{1} \\
\vdots \\
B_{n}
\end{array}\right]=\left[\begin{array}{c}
y\left(\varphi_{0}\right) \\
y\left(\varphi_{1}\right) \\
\vdots \\
y\left(\varphi_{n}\right)
\end{array}\right]
$$

Bernstein's coefficient can be acquired using Eq. (6). Then, the matching curve of the airfoil will be gained using Eqs. (1)-(3) (Liu et al., 2014).

The traditional method for choosing control points is to sample homogeneously along the horizontal ordinate.

$$
\varphi_{j}=\frac{N_{1}+j}{N_{1}+N_{2}+n}, \quad j=0,1, \cdots, n
$$

If $n=5$, the distribution of the control points on the surface of NACA 0012 is shown in Fig. 5a, and the matching error distribution is shown in Fig. 6. It is clear that the nose and tail of the airfoil have a higher error than the main body. To improve the matching accuracy of the nose and tail regions, the authors redistributed the sample points on the airfoil, and this is illustrated in Fig. 5b. The sampling method is as follows:

$$
\begin{gathered}
\varphi_{j}=0.5+0.5 \tanh \left[\lambda \cdot\left(\frac{N_{1}+j}{N_{1}+N_{2}+n}-0.5\right)\right], \\
j=0,1, \cdots, n,
\end{gathered}
$$

where $\lambda$ can be determined by optimization, and it has a different optimal value when the target is different. In this case, $\lambda=4.2$; thus, the control points were refined to be in the direction of the nose and tail. As a result, the matching error distribution is improved 
through Bernstein's coefficient obtained by this sampling method, which can be identified in Fig. 6.
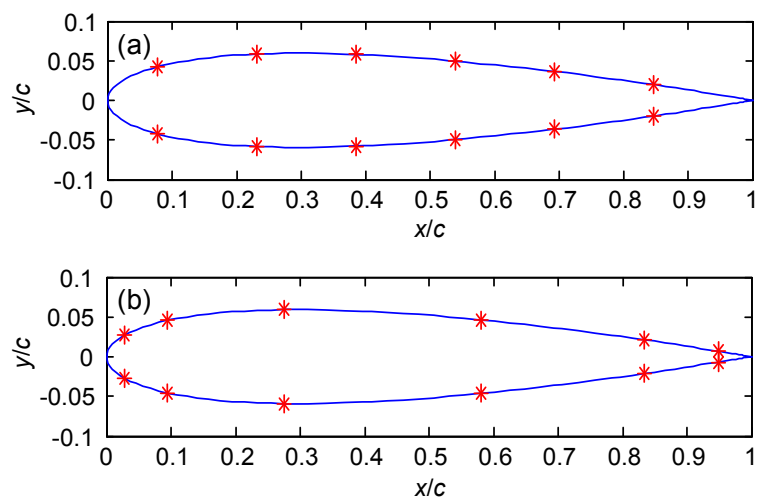

Fig. 5 Comparison between different methods to obtain control points

(a) Original method; (b) Refined method

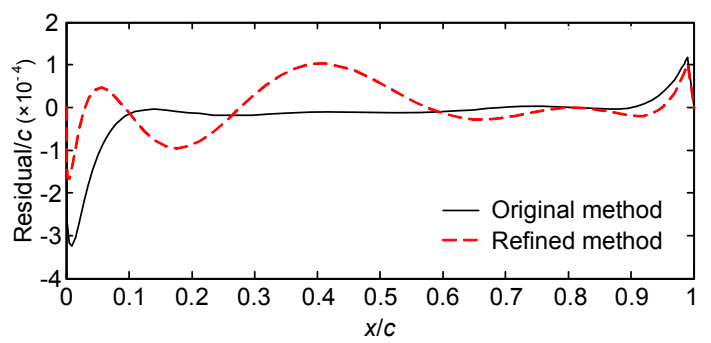

Fig. 6 Residual comparison for imitating the upper side of NACA 0012 between the original and the refined methods

The CST method has an excellent ability for parameterization; thus, it has become more and more popular for $2 \mathrm{D}$ airfoil parameterization. The authors refined the method, and obtained a higher matching accuracy. CST can also be expanded to 3D space for parameterizing aircraft. Fig. 7 demonstrates a waverider aircraft being imitated by the 3D CST method, and work in this field will be conducted in the near future.

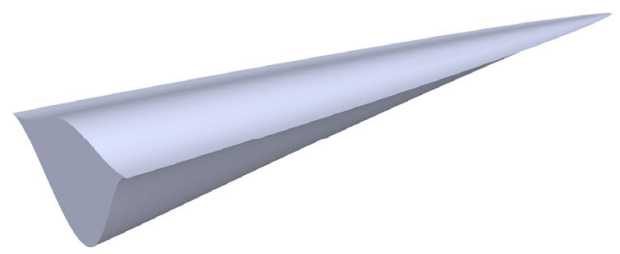

Fig. 7 Wave-rider aircraft imitation using a 3D CST method

\section{Numerical method}

NACA 0012 is a standard airfoil which is often used in experimentation and simulation; hence, plenty of reference data are available for comparison. Therefore, NACA 0012 is chosen to be the optimization target. Firstly, the independency of the grid density has been checked by the CFD method, and the results have been compared with the available experimental data in the open literature. Thus, the effectiveness of the CFD result can be proved. Qian and Wang (2011) experimentally tested the pressure distribution on the surface of NACA 0012 at low velocity, and the result has been compared with the CFD result. The computational conditions in this study are set according to the experimental conditions of Qian and Wang (2011). The main flow is set to be pressure far-field with an original speed of $8 \mathrm{~m} / \mathrm{s}$. The attack angle is $2.5^{\circ}$, while the temperature is $298 \mathrm{~K}$ and the pressure is $101325 \mathrm{~Pa}$. The boundary condition of the outlet is set to outflow, and adiabatic and no-slip conditions are assumed for the surfaces of the airfoil. The Spalart-Allmaras turbulence model is applied to the model, which was widely used in airfoil design and has been proved to be accurate (Nordanger et al., 2015), in addition to turbulent diffusion combustion in supersonic flows (Huang et al., 2012a). The equations are solved using a pressure-based (coupled) double-precision solver in Fluent Inc. (2006), and a second upwind scheme is applied to the convection terms. The SIMPLE method is employed to solve the equations. The calculation process finishes when all of the residuals converge to the order of $10^{-5}$.

The grid around the airfoil is a type of C-grid generated by the commercial software Pointwise. The flow field has a distance of 4-times chord length in front of the airfoil and a 10-times chord length from the tail to the outlet. The grid has been refined to the direction of the airfoil with the grid height of the first layer $\Delta s=0.001 \mathrm{~mm}$ to confirm Yplus $y^{+}<5$ (Fluent Inc., 2006), so that the result around it can be more precise. Fig. 8 illustrates the global and local grid distributions around the NACA 0012 airfoil.

\section{Grid independency analysis}

Smirnov et al. (2015) stated that the accumulation of stochastic error is proportional to the number 
of time steps and depends on the accuracy of the scheme and the approximation error. Three grids of different densities are generated to check the grid independency, namely coarse, moderate, and refined grids. Information for these is shown in Table 1.

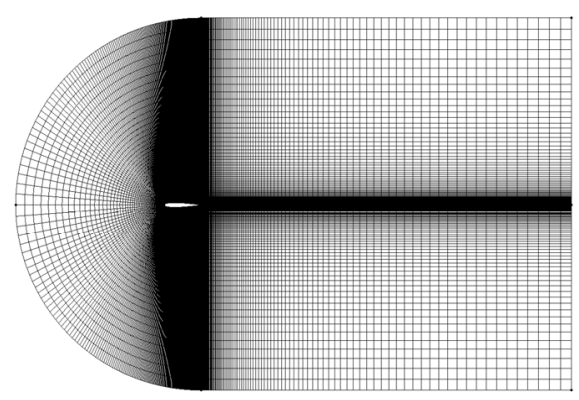

(a)

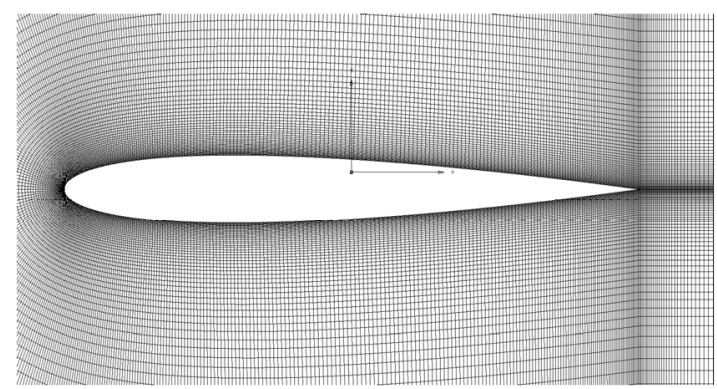

(b)

Fig. 8 Global (a) and local (b) grid distributions around NACA 0012

Table 1 Comparison of different grid densities around the NACA 0012 airfoil

\begin{tabular}{lcc}
\hline \multicolumn{1}{c}{ Grid } & Grid density & Validation metric \\
\hline Coarse & $60 \times 370$ & 0.9476 \\
Moderate & $90 \times 370$ & 0.9541 \\
Refined & $90 \times 470$ & 0.9535 \\
\hline
\end{tabular}

The CFD process is applied to all of the grids with the same computational and convergent conditions. After that, the pressure coefficient distribution on the surface of the airfoil is collected from the result, and it is then compared with the experimental data from Qian and Wang (2011). The results are shown in Fig. 9. Qian and Wang (2011) also studied the influence on the pressure distribution induced by the distance between the lower surface of the airfoil and the surface of the wind tunnel. As a result, the experimental data of the lower surface may have a few deviations from the actual number, and this may have some impact on the comparison. It can be clearly observed in Fig. 9 that the predicted results all show reasonable agreement with the experimental data. To compare the CFD result and the experimental data quantitatively, Ding et al. (2015) employed a method to transform the graphic comparison to a numerical one, which is easier to compare. The method is as follows:

$$
V=1-\frac{1}{N} \sum_{i=1}^{N} \tanh \left|\frac{y\left(x_{i}\right)-Y\left(x_{i}\right)}{Y\left(x_{i}\right)}\right|,
$$

where $y\left(x_{i}\right)$ stands for the CFD result of sample $i$ in the horizontal ordinate $x_{i}$ while $Y\left(x_{i}\right)$ is the experimental data. $V$ is the validation metric, which is better the closer it is to 1 . This value will give the quality of the CFD result when the experimental measurement error is zero.

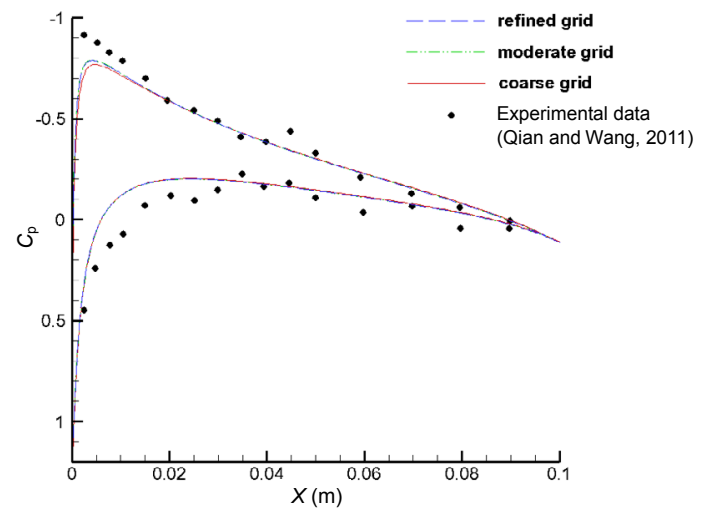

Fig. 9 Wall pressure coefficient $\left(C_{\mathrm{p}}\right)$ comparison of NACA 0012

Nevertheless, this method is not perfect because different results will be acquired when the baselines of the data are different. For example, the validation metric obtained when $Y\left(x_{i}\right)=1$ is closer to 1 than the value obtained when $Y\left(x_{i}\right)=0$, although $y\left(x_{i}\right)-Y\left(x_{i}\right)$ remains constant. To settle this problem, we modified the equation as follows:

$$
V=1-\frac{1}{N} \sum_{i=1}^{N} \tanh \left|\frac{y\left(x_{i}\right)-Y\left(x_{i}\right)}{\max \left|Y\left(x_{i}\right)\right|}\right|
$$

This new method can give a reasonable comparison result for every circumstance except $Y\left(x_{i}\right) \equiv 0$. 
Table 1 shows the validation metrics of different grid densities calculated by Eq. (10) in comparison with the experimental data. The numbers show that three grids of different densities can obtain good CFD results which fit well with the experimental data, although the moderate grid has the greatest validation metric. Consequently, the moderate grid will be used as the standard grid in the following calculation.

\section{Sampling and modeling approach}

An airfoil with better capacity in the lowvelocity condition is required in this study. The curve of NACA 0012 will be applied as the design baseline. The third-order CST method is employed to parameterize the airfoil curves upwards and downwards, and Fig. 3 has already illustrated the fitting effect. In this circumstance, eight Bernstein's coefficients in total are needed as the design variables. The lift-to-drag ratio of the airfoil for the low-velocity condition is chosen to be the optimization objective, and it is expected to be as large as possible. Bernstein's coefficient is limited to range in the zone from $70 \%$ to $130 \%$ of the baseline to prevent tortuosity of the curves, which will influence the quality of the grid. Therefore, the optimization result will be restricted in certain values, and the permission zone of Bernstein's coefficient could be enlarged in future. The optimization problem can be expressed as follows:

$$
\begin{array}{ll}
\max & C_{1} / C_{\mathrm{d}}, \\
\text { s.t. } & \left|\frac{B_{i}-b_{i}}{b_{i}}\right| \leq 0.3, \quad i \in I=\{0,1, \cdots, 7\},
\end{array}
$$

where $C_{1}$ and $C_{\mathrm{d}}$ are the lift and drag coefficients of the airfoil, respectively, and $b_{i}$ is the original Bernstein's coefficient of NACA 0012.

The computational cost will be immense with an increase in the number of design variables, especially when the samples are challenging. It is urgent to acquire an accurate optimization result when the number of samples has been decreased. The surrogate model will construct a mathematical model which will imitate the original physical model with high precision using few samples. The optimization process applied on the surrogate model will dramatically cut down the computational cost. In recent years, finding a surrogate model with high accuracy and which needs only a small number of samples has been of great concern, and many new models have appeared including the kriging model (Namura et al., 2015), the radial basis function model (Wu Z.Y. et al., 2015), the artificial neural network model, the multiple output Gaussian process (MOGP) (Liu et al., 2014), and so on. Among all of the surrogate models, the polynomial-based response surface model (P-RSM) is the most mature one, which has been employed successfully in aerodynamic design and reverse design (Wu X.J. et al., 2015). Therefore, the fourth-order polynomial response surface model with cross terms has been applied in this study.

To guarantee the predictive accuracy of the surrogate model while reducing the number of samples, a reasonable sampling method is of great importance for enhancing the design efficiency. Latin hypercube sampling (LHS) was originally presented by McKay et al. (1979), and is a type of space-filling design technique (Huang et al., 2012b). The sampling method is:

$$
x_{j}^{(i)}=\frac{\pi_{j}^{(i)}+U_{j}^{(i)}}{N},
$$

where $i$ represents the serial number of the sample, while $j$ represents the serial number of the design variable. $U$ is a random number in the range from 0 to $1 ; \pi$ is a random permutation of $0,1, \ldots, N-1$, while $N$ is the total number of the samples. As a result, $\pi_{j}^{(i)}$ and $U_{j}^{(i)}$ determine the local area and the specific coordinate in this area of $x_{j}^{(i)}$. This sampling method can assure that there are subintervals which have the same number as the samples in every dimension. Moreover, there will be one and only one sample in every subinterval. The application of a random number may ensure that the sample will distribute randomly within the subinterval. On the other hand, because of the random number, the samples are likely to aggregate in a certain area, and thus they cannot distribute uniformly. The uniform LHS is a modification of the LHS, which can obtain the optimum design strategy by applying several optimization techniques on the original LHS method (Shi et al., 2012). This method will avoid a uniform distribution of the samples, and thus is applied in this study. 


\section{Optimization method}

It is a significant consideration to choose a good optimization method to get the best design results, and the quality of the optimization method will influence the credibility and efficiency of the process. CFD solvers are typically combined with numerical optimization methods, in particular gradient-based and non-gradient-based methods. Non-gradient-based numerical optimization methods, such as genetic algorithms, are generally not as efficient as gradientbased methods. However, gradient-based methods require a design space free from discontinuity as the derivatives have to be recalculated as the search progresses (Nejat et al., 2014). Further, when a problem is multimodal, gradient-based methods will fail to find solutions beyond the local best result close to the start point. Therefore, to compare the efficiency of gradient-based and non-gradient-based methods, the multi-island genetic algorithm (MIGA), nonlinear programming by quadratic Lagrangian (NLPQL), and a combination of them were utilized in this study (Fig. 10). Genetic algorithms are classical stochastic optimization algorithms inspired by evolutionary analogy. In MIGA, the population is divided into several sub-populations staying on isolated "islands", whereas traditional genetic algorithm operations are performed on each sub-population separately. All traditional genetic operations are performed separately on each sub-population. Some individuals are then selected from each island and migrated to different islands periodically. This operation is called 'migration'. Thus, MIGA can prevent the problem of being 'premature' by maintaining the diversity of the population. In addition, the calculation speed of MIGA can be faster than those of traditional genetic algorithms (Zhao et al., 2015). NLPQL is a sequential quadratic programming (SQP) method which can solve problems by continuous smoothing. The Hessian of the Lagrange function is used to make a quadratic approximation of the Lagrange function (Chen and Lv, 2014). When solving constrained nonlinear mathematical problems, the NLPQL algorithm shows stability, rapid convergence, and the capacity to seek globally optimal solutions. As the NLPQL can quickly determine the local optimal solution near the starting point, it can reduce computing time during the optimization process (Li et al., 2014). It is offered in the commercial software ISIGHT. ISIGHT is an excellent optimization solving method. Its integration ability makes it an efficient tool for combining different software and programs together to achieve automation of sampling, modeling, and optimization. The samples we obtain from it can also be exported so that they can be processed in other modeling and optimization programs with improved performance.

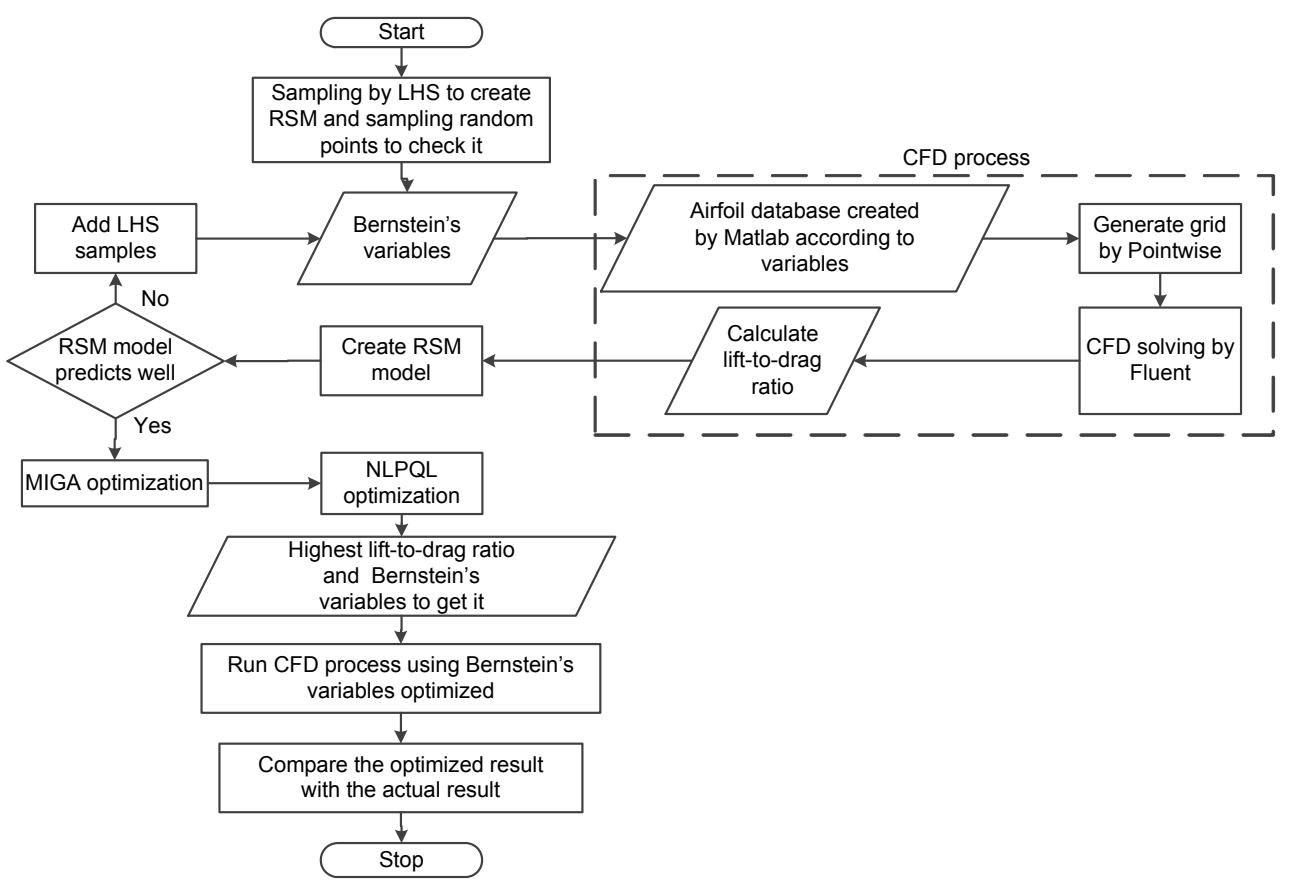

Fig. 10 Schematic diagram of sampling, modeling, and optimization 
MIGA is a global optimization method, which can find the result next to the best one. NLPQL is a local optimization method that can explore the best result around the start point. The combination of MIGA and NLPQL involves using MIGA to obtain the expected area and then following that with NLPQL to obtain the best point. A flow chart describing this method using ISIGHT is given in Fig. 11. The Matlab item will generate the database of airfoils according to the variables generated by the uniform LHS. Pointwise \& Fluent is a batch file for accomplishing meshing and CFD solving progress, and the lift force and drag force of the airfoil will be exported. After that, the lift and drag will be read to ISIGHT, and the lift-to-drag ratio will be calculated. After 122 rounds of this process, the RSM will be established, and another 61 rounds of this progress will run after that to verify the effectiveness of this model. Finally, MIGA followed by NPLQL will explore the best point to maximize the lift-to-drag ratio.

\section{Results and discussion}

Some 122 variable samples were generated by the uniform LHS to establish the response surface model, while another 61 variable samples obtained randomly were utilized to check the model. Fig. 12 shows the comparison of lift-to-drag ratio of the random samples with the predicted value by RSM as the horizontal axis and the CFD result as the vertical axis. The solid line is the central line with equal horizontal ordinate and vertical ordinate, while the points stand for the results of the 61 samples mentioned above. It shows that the results predicted by RSM meet well with the actual results gained from the CFD method, as the points are spread precisely along the solid line. As a result, it can be believed that the polynomial-based response model we use is effective and that it can be used for the optimization process instead of using the CFD process.

The MIGA, NLPQL, and MIGA-based NLPQL method were utilized to optimize the RSM model established previously. Before executing the MIGA method, the number of generations, the number of islands, and the number of individuals on each island were all set to 10 . The number of interval generations of migration was set to 5 . The crossover rate was set to 1 , while the rates of migration and mutation were

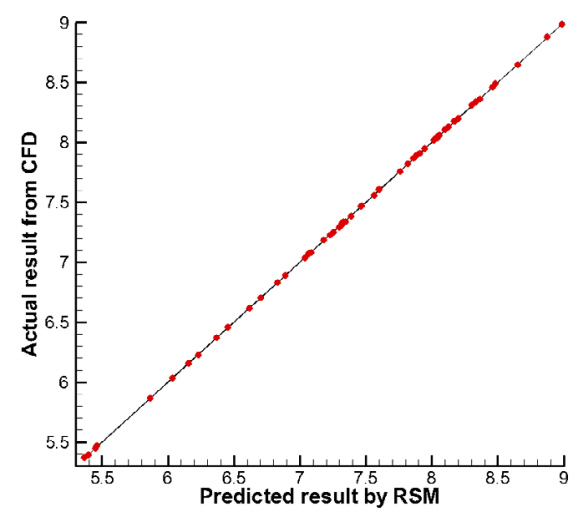

Fig. 12 Comparison of response value calculated and predicted by RSM

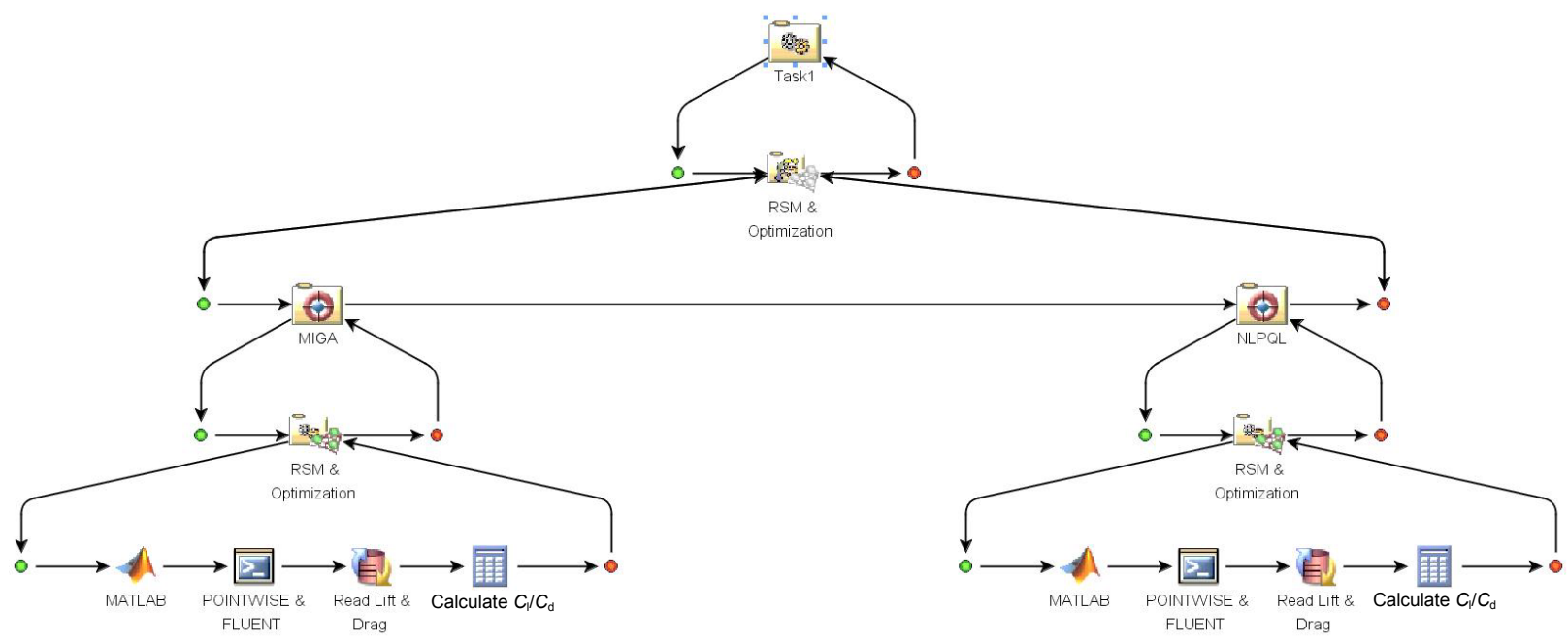

Fig. 11 Processing flow of the combination of MIGA and NLPQL in ISIGHT 
both 0.01 . In contrast, when NLPQL was used, the maximum iterations were 40 , and the termination accuracy was $10^{-6}$, while the relative step size was set to 0.001 . The parameters of each method were all kept the same when MIGA-based NLPQL was utilized.

The iteration histories of the lift-to-drag ratio obtained by the three optimization methods, namely MIGA only, NLPQL only, and the combination of MIGA and NLPQL, are shown in Fig. 13, and the results of the comparison of different optimization
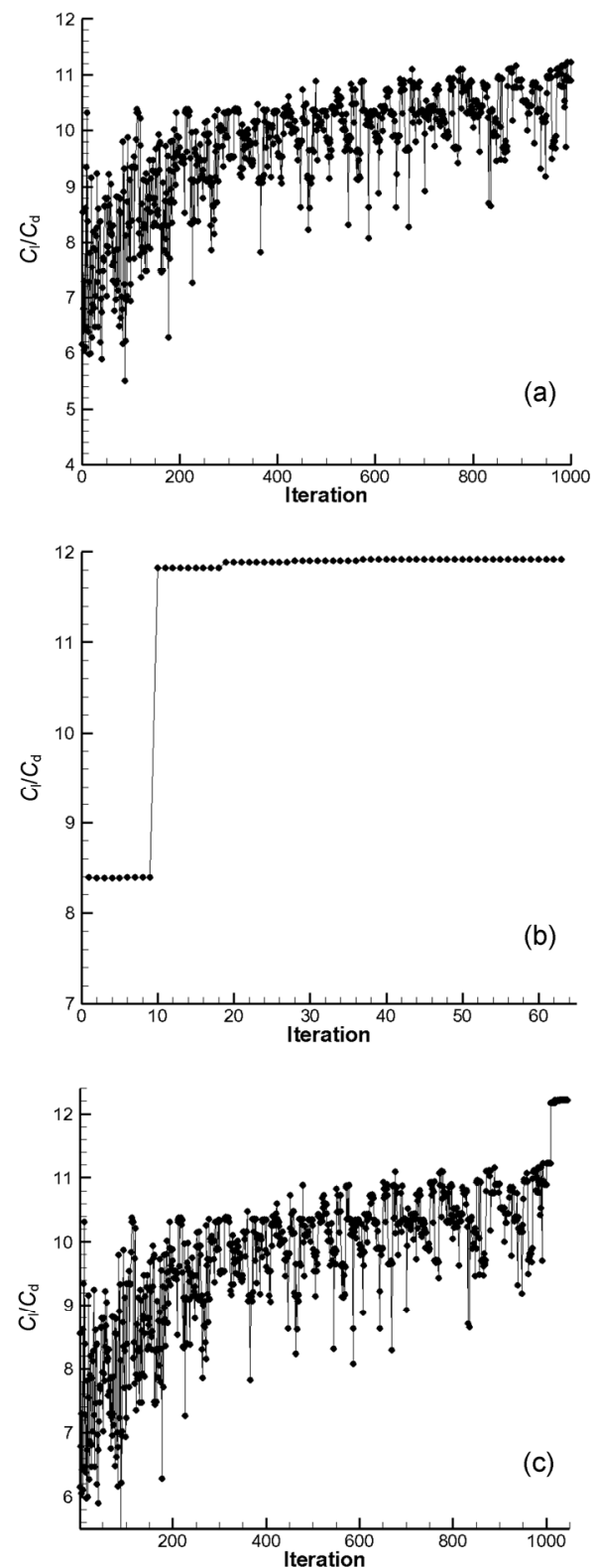

Fig. 13 Optimization history of three optimization methods: (a) MIGA only; (b) NLPQL only; (c) combination of MIGA and NLPQL methods are given in Table 2. Bernstein's variables at both the lower side $(\mathrm{Bl})$ and the upper side $(\mathrm{Bu})$ of the airfoil were all optimized based on the RSM model, and the highest lift-to-drag ratios were predicted by the RSM. After that, the real lift-to-drag ratios based on Bernstein's variables were calculated using the CFD method; thus, they can be compared with the original value and predicted results. The result illustrates that the RSM model predicts credible results because the lift-to-drag ratio predicted meets well with the real results. Compared with the original airfoil, the lift-to-drag ratio optimized by the MIGA method increased by $49.43 \%$, while the lift-to-drag ratio optimized by the NLPQL method increased by $59.15 \%$. However, neither of the results gained are optimal. Based on the result obtained from MIGA, NLPQL improved the lift-to-drag ratio by $62.32 \%$. It shows that MIGA-based NLPQL can achieve an improved optimization result compared with the single optimization method. To compare the computational cost of the three methods, computational time and iteration number are also given in Table 2, from which we find that the MIGA-based NLPQL method costs more time and iterations than the other two optimization methods. However, based on the response surface model established previously, the computational cost of the optimization process has been minimized dramatically; thus, it can be neglected from the complete flow path.

Figs. 14 and 15 show the airfoils optimized by three optimization methods and the $C_{\mathrm{p}}$ distribution on the airfoil, respectively. We can conclude that although the airfoils look alike, their capability may differ a lot. Therefore, an accurate parametric method for describing airfoils and a good optimization method to improve them are very significant for airfoil design.

Since we have known that the airfoil with the highest lift-to-drag ratio was gained by the MIGAbased NLPQL method, line contour graphs of pressure coefficient in the flow-field around the airfoil are compared in Fig. 16. From the comparison we find that, under the attack angle of $2.5^{\circ}$, the main lift of the airfoil comes from the suck force on the upper side. Before optimization, the area of negative pressure on the upper side of NACA 0012 is small, but the area is enlarged after optimization. Moreover, the underside of the NACA 0012 suffers negative pressure, 
Table 2 Result comparison of different optimization methods

\begin{tabular}{lcccccccccccc}
\hline \multicolumn{1}{c}{ Method } & $\mathrm{Bl}_{0}$ & $\mathrm{Bl}_{1}$ & $\mathrm{Bl}_{2}$ & $\mathrm{Bl}_{3}$ & $\mathrm{Bu}_{0}$ & $\mathrm{Bu}_{1}$ & $\mathrm{Bu}_{2}$ & $\mathrm{Bu}_{3}$ & $\begin{array}{c}\text { Predicted } \\
C_{\mathrm{l}} / C_{\mathrm{d}}\end{array}$ & $\begin{array}{c}\mathrm{Actual} \\
C_{\mathrm{l}} / C_{\mathrm{d}}\end{array}$ & $\begin{array}{c}T_{\mathrm{c}} \\
(\mathrm{s})\end{array}$ & $N_{\mathrm{i}}$ \\
\hline $\begin{array}{l}\text { Original } \\
\text { Multi-island } \\
\text { GA only }\end{array}$ & 0.16898 & 0.15133 & 0.13829 & 0.13912 & 0.16898 & 0.15133 & 0.13829 & 0.13912 & - & 7.45761 & - & - \\
NLPQL only & 0.11829 & 0.10593 & 0.09680 & 0.09738 & 0.15709 & 0.19673 & 0.17980 & 0.15289 & 11.91620 & 11.86880 & 1 & 63 \\
$\begin{array}{c}\text { Multi-island } \\
\text { GA+NLPQL }\end{array}$ & 0.11829 & 0.10593 & 0.09680 & 0.09738 & 0.15227 & 0.19673 & 0.17980 & 0.18086 & 12.21420 & 12.10500 & 7 & 1046 \\
\hline
\end{tabular}

$T_{\mathrm{c}}$ : computational time; $N_{\mathrm{i}}$ : iteration number

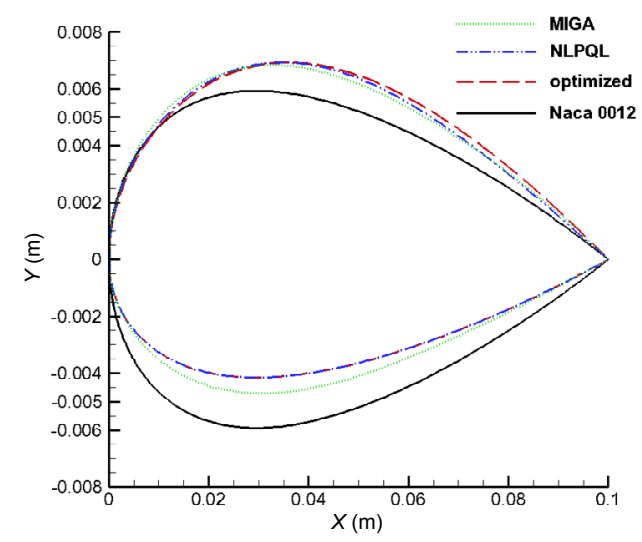

Fig. 14 Airfoils optimized by three optimization methods

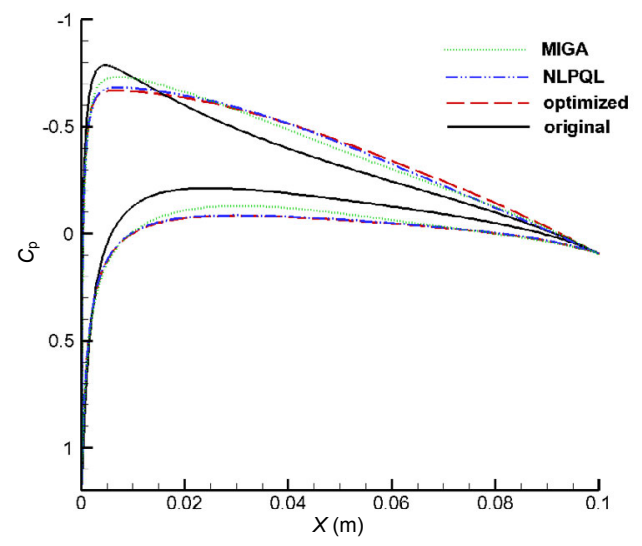

Fig. 15 Comparison of wall pressure coefficient distribution of airfoils optimized by three methods

which may counteract the lift force generated by the upper surface. However, this problem is settled after optimization. As a result, the lift force of the airfoil is improved.

From the optimization record, we find that the drag force of the airfoil decreased by $6 \%$ in total, the main contribution to this being pressure drag, which

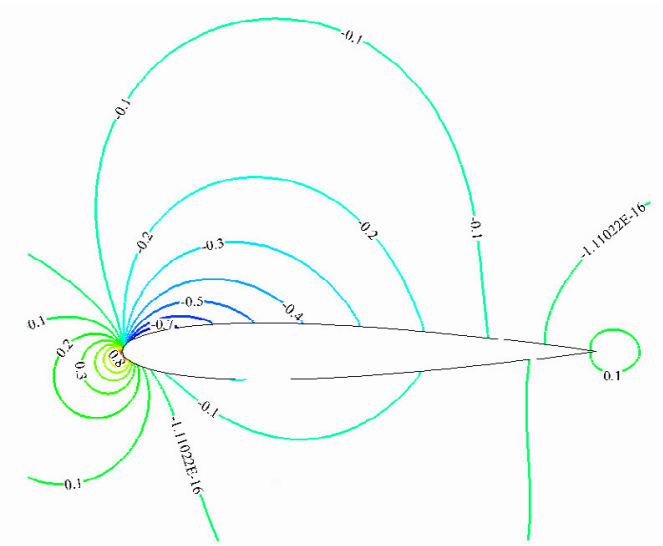

(a)

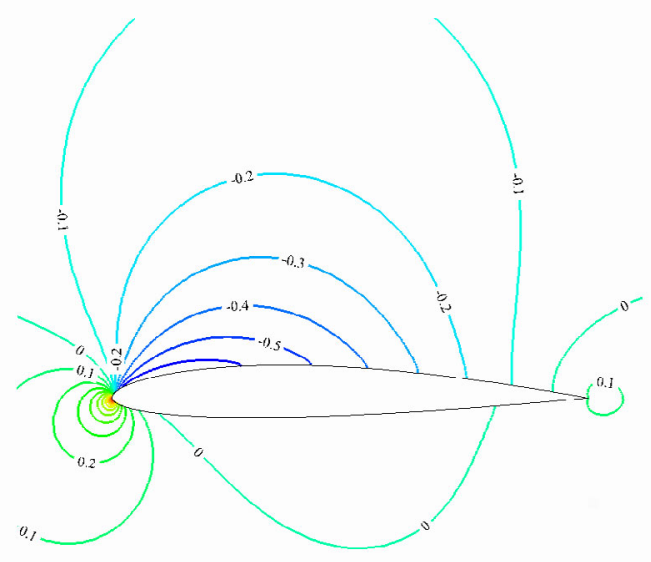

(b)

Fig. 16 Line contour graphs of pressure coefficient in the flow-field around the airfoil: (a) NACA 0012; (b) optimized airfoil

cannot be distinguished easily from the distribution of the pressure coefficient. Therefore, the wall-shearstress distribution along the airfoil is illustrated in Fig. 17. It shows that the distribution of wall-shearstress does not change a lot after optimization, which 
implies that the viscous force is not the main reason to decrease the drag on the airfoil. Thus, the optimization does not change the viscous force a lot but boosts the lift-to-drag ratio by improving the pressure distribution on the airfoil.

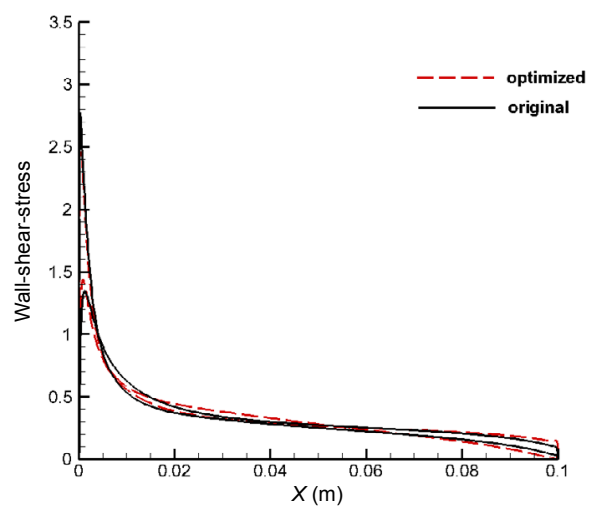

Fig. 17 Distribution of wall-shear-stress on the airfoil

To check the performance of the new airfoil we obtained, the CFD method is utilized to test the lift-to-drag ratio under different attack angles, and the result is compared in Fig. 18. NACA 0012 is a symmetrical airfoil, and thus the lift-to-drag ratio is 0 when the attack angle is 0 . It seems that the lift-todrag ratio is linear to the attack angle in a small area next to 0 , and the peak value occurs at around $7^{\circ}$. After that, the value decreases because the airfoil will suffer stall problems at high attack angles. In contrast, the lift-to-drag ratio optimized is greatly improved for a range in attack angle of $-2.5^{\circ}$ to $10^{\circ}$. When the flow passes the airfoil with no angle, the ratio can still reach 4.44. Indeed, a 0 lift-to-drag ratio point moves to a negative attack angle of $-1.3^{\circ}$. However, the stall characteristics are not improved after optimization, and this could be another optimization objective for future work. This is a multi-objective design optimization problem, and it should be solved using the multi-objective design optimization approach and data mining method (Huang, 2014).

Fig. 18 shows the similarity between the two airfoils, which may cause confusion about whether the optimized airfoil is the original one rotated by a particular angle. The comparison of pressure coefficient distribution when the lift-to-ratio is 0 shown in Fig. 19 may give the answer. It is clear that the original airfoil has a symmetric configuration, and thus the pressure on the upper surface is equal to that on the lower surface. However, the airfoil after optimization shows a different reason why the lift-to-drag ratio is 0 . The upper surface has a higher pressure distribution in the nose area while the pressure in the body and tail area is smaller than the lower surface. This proves that the optimized airfoil is not symmetrical, and that the configuration has nothing in common with the original one.

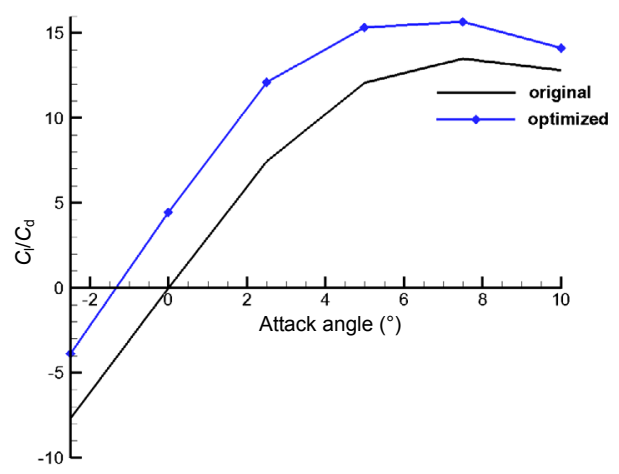

Fig. 18 Lift-to-drag ratio comparison

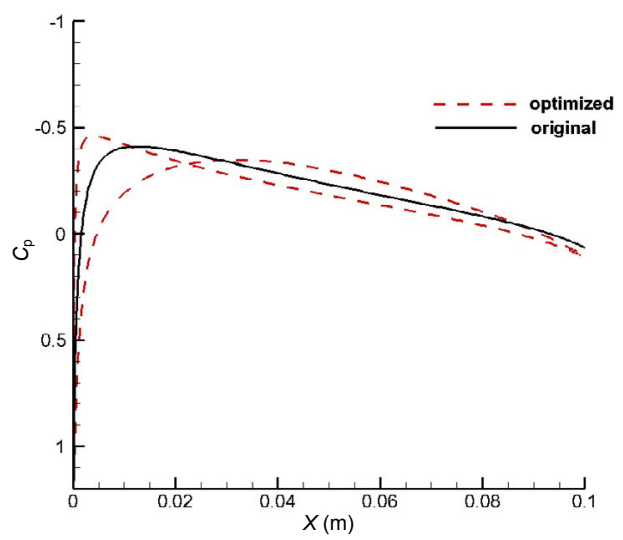

Fig. 19 Pressure coefficient distribution comparison when the lift-to-drag ratio is 0

\section{Conclusions}

In this paper, parameterization methods of airfoil have been compared and a numerical method has been utilized to optimize the airfoil with better aerodynamic performance based on the response surface model. The results show that:

1. The CST method is an excellent parametric approach, and it has been modified by redistributing 
the control points of the shape function in this paper, which allows a better definition of the nose and tail areas of the airfoil. In comparison with the polynomial, the CST method has a higher imitation accuracy with fewer design variables, which is beneficial for the optimization process.

2 . The nonlinear programming by quadratic Lagrangian optimization method is utilized after the multi-island genetic algorithm, which will search for the airfoil with the highest lift-to-drag ratio. Comparison of the optimization results shows that a combination of the two optimization methods can achieve better results than the methods used separately. Although it will cost more computational time and use more steps, the effect can be neglected from the whole flow path, because the response surface model is established to replace the CFD process and the computational cost has been reduced dramatically.

After optimization, the result shows that the lift-to-drag ratio has improved a lot by enhancing the lift force and decreasing the drag force. However, the stall characteristics of the airfoil have not been improved, and this should be investigated in the near future.

\section{References}

Chen, Y., Lv, L., 2014. The multi-objective optimization of combustion chamber of DI diesel engine by NLPQL algorithm. Applied Thermal Engineering, 73(1):1332-1339. http://dx.doi.org/10.1016/j.applthermaleng.2014.09.028

Ding, F., Shen, C.B., Huang, W., et al., 2015. Numerical validation and back-pressure effect on internal compression flows of typical supersonic inlet. The Aeronautical Journal, 119(1215):631-645. http://dx.doi.org/10.1017/S0001924000010721

Fluent Inc., 2006. Fluent 6.3 User's Guide. Fluent Inc., Lebanon.

Huang, W., 2014. Design exploration of three-dimensional transverse jet in a supersonic crossflow based on data mining and multi-objective design optimization approaches. International Journal of Hydrogen Energy, 39(8):3914-3925.

http://dx.doi.org/10.1016/j.ijhydene.2013.12.129

Huang, W., 2015. A survey of drag and heat reduction in supersonic flows by a counterflowing jet and its combinations. Journal of Zhejiang University-SCIENCE A (Applied Physics \& Engineering), 16(7):551-561. http://dx.doi.org/10.1631/jzus.A1500021

Huang, W., Pourkashanian, M., Ma, L., et al., 2012a. Effect of geometric parameters on the drag of the cavity flameholder based on the variance analysis method. Aerospace Science and Technology, 21(1):24-30. http://dx.doi.org/10.1016/j.ast.2011.04.009

Huang, W., Wang, Z.G., Li, S.B., et al., 2012b. Influences of $\mathrm{H}_{2} \mathrm{O}$ mass fraction and chemical kinetics mechanism on the turbulent diffusion combustion of $\mathrm{H}_{2}-\mathrm{O}_{2}$ in supersonic flows. Acta Astronautica, 76:51-59.

http://dx.doi.org/10.1016/j.actaastro.2012.02.017

Huang, W., Li, S.B., Liu, J., et al., 2012c. Investigation on high angle of attack characteristics of hypersonic space vehicle. Science China Technological Sciences, 55(5):1437-1442. http://dx.doi.org/10.1007/s11431-012-4760-6

Kevin, L., David, M., 2009. A surface parameterization method for airfoil optimization and high lift 2D geometries utilizing the CST methodology. 47th AIAA Aerospace Sciences Meeting Including the New Horizons Forum and Aerospace Exposition, Orlando, Florida, USA. http://dx.doi.org/10.2514/6.2009-1461

Kulfan, B.M., 2007. A universal parametric geometry representation method-"CST". 45th AIAA Aerospace Meeting \& Exhibit, Reno, Nevada, USA. http://dx.doi.org/10.2514/6.2007-62

Li, F., Qin, Y., Pang, Z., et al., 2014. Design and optimization of PSD housing using a MIGA-NLPQL. Strojniški vestnik - Journal of Mechanical Engineering, 60(7-8):525-535. http://dx.doi.org/10.5545/sv-jme.2013.1492

Liu, X., Zhu, Q., Lu, H., 2014. Modeling multiresponse surfaces for airfoil design with multiple-output-Gaussianprocess regression. Journal of Aircraft, 51(3):740-747. http://dx.doi.org/10.2514/1.C032465

Luo, J.N., Lu, W.X., 2014. Comparison of surrogate models with different methods in groundwater remediation process. Journal of Earth System Science, 123(7):1579-1589. http://dx.doi.org/10.1007/s12040-014-0494-0

Ma, Y., Yang, T., Feng, Z., et al., 2015. Hypersonic lifting body aerodynamic shape optimization based on the multiobjective evolutionary algorithm based on decomposition. Proceedings of the Institution of Mechanical Engineers, Part G: Journal of Aerospace Engineering, 229(7):12461266.

http://dx.doi.org/10.1177/0954410014548699

McKay, M.D., Beckman, R.J., Conover, W.J., 1979. A comparison of three methods for selecting values of input variables in the analysis of output from a computer code. Technometrics, 21(2):239-245. http://dx.doi.org/10.2307/1268522

Morris, C.C., Allison, D.L., Schetz, J.A., et al., 2014. Parametric geometry model for design studies of tailless supersonic aircraft. Journal of Aircraft, 51(5):1455-1466. http://dx.doi.org/10.2514/1.C032340

Namura, N., Shimoyama, K., Obayashi, S., 2015. Kriging surrogate model enhanced by coordinate transformation of design space based on eigenvalue decomposition. In: Gaspar-Cunha, A., Antunes, C.H., Coello, C.C. (Eds.), Evolutionary Multi-criterion Optimization. Springer International Publishing, Switzerland, p.321-335. http://dx.doi.org/10.1007/978-3-319-15934-8_22

Nejat, A., Mirzabeygi, P., Panahi, M.S., 2014. Airfoil shape 
optimization using improved multiobjective territorial particle swarm algorithm with the objective of improving stall characteristics. Structural \& Multidisciplinary Optimization, 49(6):953-967.

http://dx.doi.org/10.1007/s00158-013-1025-3

Nordanger, K., Holdahl, R., Kvamsdal, T., et al., 2015. Simulation of airflow past a 2D NACA0015 airfoil using an isogeometric incompressible Navier-Stokes solver with the Spalart-Allmaras turbulence model. Computer Methods in Applied Mechanics and Engineering, 290: 183-208.

http://dx.doi.org/10.1016/j.cma.2015.02.030

Qian, J.L., Wang, D.F., 2011. Experimental and numerical study on the aerodynamics characteristics of an airfoil adjacent to a ground. Chinese Journal of Hydrodynamics, 26(2):150-156 (in Chinese). http://dx.doi.org/10.3969/j.issn1000-4874.2011.02.003

Shi, L., Yang, R.J., Zhu, P., 2012. A method for selecting surrogate models in crashworthiness optimization. Structural and Multidisciplinary Optimization, 46(2):159-170. http://dx.doi.org/10.1007/s00158-012-0760-1

Smirnov, N.N., Betelin, V.B., Nikitin, V.F., et al., 2015. Accumulation of errors in numerical simulations of chemically reacting gas dynamics. Acta Astronautica, 117: 338-355. http://dx.doi.org/10.1016/j.actaastro.2015.08.013

Sobieczky, H., 1999. Parametric airfoils and wings. In: Fujii, K., Dulikravich, G.S. (Eds.), Recent Development of Aerodynamic Design Methodologies. Vieweg+Teubner Verlag, p.71-87. http://dx.doi.org/10.1007/978-3-322-89952-1_4

Straathof, M.H., van Tooren, M.J.L., 2011. Extension to the class-shape-transformation method based on B-splines. AIAA Journal, 49(4):780-790. http://dx.doi.org/10.2514/1.J050706

Straathof, M.H., van Tooren, M.J.L., 2012. Adjoint optimization of a wing using the class-shape-refinementtransformation method. Journal of Aircraft, 49(4):10911100 . http://dx.doi.org/10.2514/1.C031594

Su, H., Gu, L., Gong, C., 2015. Research on geometry modeling method based on three-dimensional CST parameterization technology. 16th AIAA/ISSMO Multidisciplinary Analysis and Optimization Conference, Dallas, USA. http://dx.doi.org/10.2514/6.2015-3241

Wu, X.J., Zhang, W.W., Xiao, H., et al., 2015. A robust aerodynamic design for airfoil based on response surface method. Engineering Mechanics, 32(2):250-256 (in Chinese). http://dx.doi.org/10.6052/j.issn.1000-4750.2013.09.0847

Wu, Z.Y., Chen, Y., Yao, W., et al., 2015. A RBF neural network modeling method based on sensitivity analysis and Pareto law. 11th World Congress on Structural and Mul- tidisciplinary Optimisation, Sydney, Australia.

Yu, J.Z., Saeed, F., Paraschivoiu, I., 2003. Efficient optimized airfoil parameterization. 41st AIAA Aerospace Meeting \& Exhibit, Reno, Nevada, USA.

http://dx.doi.org/10.2514/6.2003-725

Zhang, D.H., Xi, S., Tian, D., 2014. Geometry control ability evaluation of classical airfoil parametric method. $A d$ vances in Aeronautical Science and Engineering, 5(3): 281-288 (in Chinese).

Zhao, D.J., Wang, Y.K., Cao, W.W., et al., 2015. Optimization of suction control on an airfoil using multi-island genetic algorithm. Procedia Engineering, 99:696-702. http://dx.doi.org/10.1016/j.proeng.2014.12.591

\section{中文概要}

\section{题 目: 基于数值计算方法的翼型参数化、建模与优化} 研究

目 的: 1. 比较并改善翼型参数化方法, 获得设计变量少、 拟合精度高的参数化方法; 2 . 在参数化的基础上 利用数值模拟的方法获取翼型流场参数, 优化并 获得特定条件下升阻比最大的翼型。

创新点: 1. 通过与多项式拟合方法的对比证明了类别/形状 函数转换 (CST) 法在翼型拟合方面的优越性, 并通过调整控制点分布, 在不增加设计变量的基 础上改善了 CST 方法; 2. 通过建立响应面模型, 利用多岛遗传算法与非线性序列二次规划法相 结合的方式获得了更好的翼型优化效果。

方 法: 1. 利用修饰后的 CST 法对翼型进行参数化拟合与 设计, 并通过与二项式拟合法比较来验证其优越 性; 2. 通过数值方法对翼型周围流场进行计算并 与实验结果对比, 获得精确计算气动参数的仿真 条件; 3. 通过拉丁超立方采样获得设计变量, 建 立设计变量与翼型升阻比之间的响应面模型, 通 过多岛遗传算法与非线性序列二次规划法的结 合和优化, 得到一定条件下升阻比最大的翼型。

结 论: 1. CST 法是一种优秀的参数化方法, 本文的优化 改善了形状函数控制点选取法则, 使其对翼型头 部和尾部的描述更加精确; 与多项式相比, CST 法可以通过更少的设计变量得到更高的拟合精 度。2. 基于多岛遗传算法的非线性序列二次规划 法在本文中用以优化翼型使其具有更高升阻比。 优化前后翼型的比较显示, 两种优化方法的结合 可以得到比单独使用各优化方法更好的结果。

关键词: 类别/形状函数转换; 参数化; 数值仿真; 响应面 模型; 优化; 翼型设计 